InnOvaciOnes de NegOciOs 16(31): 1-25

(c) 2019 UANL, Impreso en México (ISSN: 2007-1191)

Recepción: 23 Julio de 2018. Aceptación: 27 Agosto 2018

\title{
El papel de Innovación y el cambio tecnológico desde una visión económica (The role of innovation and technological change from an economic perspective)
}

\author{
José Antonio Velázquez Juárez \\ Universidad Autónoma de Querétaro \\ jose_antonio_velazquez@hotmail.com
}

\begin{abstract}
In this research, the processes that the market and the decisions of a public management are analyzed, and can have an influence over the creation of technological change pattern, which it leads to give an explanation about the reasons that can make grow the current or potential production of a national economy in the long term. To this end, several topics related with production factors, technological change, innovation, economic cycles, economic growth and accumulation of capital are included, which have a big repercussion from the economic perspective to explain the importance of technological progress over the economic activities at a national level. Additionally, this article pretends to explain from a macroeconomic perspective, the impact that innovation and technological change generates, like an amount of activities that are developed from a compilation of multiple theoretical contributions from the area of innovation and technological change.
\end{abstract}

Key words: Innovation, Technological Change, Macroeconomics, Technology.

JEL: 031, 033.

Resumen. En este trabajo, se analizan los diferentes procesos que el mercado y las decisiones de las administraciones públicas influyen en la creación de patrones de cambio tecnológico, y que conlleva a explicar las razones que pueden hacen crecer la producción actual o potencial de una economía nacional en el largo plazo. Para ello, se consideran tópicos relacionados con factores de producción, cambio tecnológico, innovación, ciclos económicos, crecimiento económico y acumulación de capital, que tienen una gran repercusión desde la visión económica para explicar la importancia del

\section{El papel de innovación y el cambio tecnológico}


progreso tecnológico en las actividades económicas a nivel nacional. Adicionalmente, en este artículo se busca explicar desde el ángulo de estudio del análisis macroeconómico el impacto que genera la innovación y el cambio tecnológico, como una serie de actividades que se desarrollan desde una compilación de diversas aportaciones teóricas provenientes del área de la innovación y del cambio tecnológico.

Palabras clave: Innovación, Cambio Tecnológico, Economía, Tecnología.

\section{Introducción}

La acumulación de capital físico y humano al desarrollo de actividades económicas han tenido una amplia contribución al crecimiento económico, mediante la generación masiva de bienes, pero la aportación de la innovación y el progreso tecnológico, es decir el descubrimiento y la aplicación de nuevas tecnologías ha tenido un mayor impacto, ya que esta actividad implica el descubrimiento de nuevas formas de aprovechar los factores de producción disponibles, mediante la investigación formal, los programas de desarrollo y el ensayo y error informal (Parkin y Loria, 2010).

Sin embargo, con la intención de aprovechar las bondades que otorga el cambio tecnológico, se requiere contar con un mayor capital que se traduzca en una mayor actividad laboral, además de que algunas de las tecnologías fundamentales tales como el lenguaje, la escritura y las matemáticas representan un componente primordial del capital humano, aunque por otro lado, muchas tecnologías están incorporadas en el capital físico, aunque para aprovechar la evolución de esas tecnologías, se tuvieron que reemplazar los carruajes y caballos por

\section{Velázquez Juárez, J.}


artefactos más sofisticados tales como los motores de combustión interna, así como el uso de tocadiscos fue sustituido por el uso de dispositivos de música digital como Spotify y Itunes.

Al mismo tiempo que se incentiva la formación de capital a través del cambio tecnológico, es muy común llevar a cabo este proceso mediante la ayuda del exterior y de las inversiones extranjeras y de esta forma se busca incrementar al máximo la difusión de los conocimientos tecnológicos necesarios, donde se observa que la ayuda extranjera se dirige hacia los sectores de infraestructura física y social, esto es, generación de proyectos de inversiones públicas, tales como carreteras, hospitales e infraestructura educativa (Clement y Pool, 1997).

Con el impulso que se genera por la aplicación de avances tecnológicos provenientes de las naciones industrializadas que otorgan el capital físico mediante procesos de transferencia tecnológica, se busca que las compañías o sectores industriales que sean receptores de esa tecnología, puedan contar con la habilidad de enfrentar con un mayor espectro de retos contemporáneos, el hecho de que la tecnología transforma al mundo e influye en las vidas de las personas, considerando costos, calidad y difusión.

Uno de los principales elementos que se ve modificado por la acumulación de capital y el cambio tecnológico es el crecimiento económico, ya que se han desarrollado diferentes modelos y teorías que facilitan estudiar el impacto que tiene el progreso tecnológico en la generación de bienes y en las actividades económicas que realizan los diferentes sectores productivos e industriales, privilegiando

\section{El papel de innovación y el cambio tecnológico}


principalmente las aportaciones de (Parkin y Loria, 2010; Samuelson Nordhaus, Dieck y Salazar, 2001).

Con la finalidad de comprender la forma en que influye en la economía la acumulación de capital y el cambio tecnológico, se requiere estudiar inicialmente el concepto de la teoría clásica del crecimiento, que considera que el crecimiento del PIB real per cápita se da de manera temporal y tiende a incrementarse por encima del nivel de subsistencia; pero las diferentes modificaciones provenientes de cambios poblacionales y demográficos modifican los niveles esperados de crecimiento económico. Sin embargo, se debe tomar en cuenta el valor que tiene actualmente la innovación tecnológica y la inversión de capital, ya que la tierra no se consideró como un factor limitante de la producción, sino que la acumulación de capital y la generación de nuevas tecnologías empezaron a ser una fuerza dominante en las actividades económicas e industriales, especialmente desde la Revolución industrial, donde se generaron mejoras notables en equipos de trabajo, medios de transporte y manejo de metales y materias primas, además de la creación de nuevas industrias alrededor del teléfono, el automóvil y la energía eléctrica.

\section{Marco teórico}

Acumulación de capital y productividad

A diferencia de la teoría clásica del crecimiento económico, Samuelson y Nordhaus (2006) sostiene que el modelo neoclásico para el crecimiento económico explica que el crecimiento del trabajo se ve

Velázquez Juárez, J. 
determinado por fuerzas ajenas a la economía y los principales factores de producción que considera este modelo son el capital y el cambio tecnológico, donde el primero consiste en los bienes duraderos producidos que se utilizan para hacer otros bienes de consumo inmediato, mientras que el segundo se enfoca en desarrollar avances logrados en los procesos de producción y en la introducción de nuevos y mejores bienes y servicios (Samuelson, Nordhaus, 2006).

Asimismo, el capital llega a intensificar su uso en el momento en el que crece más que el capital humano disponible y en ausencia de un cambio tecnológico notorio, la intensificación de capital eleva la producción por trabajador, el producto marginal del trabajo y los salarios; aunque también el capital empieza a mostrar rendimientos decrecientes, esto es, que al aumentar de forma sucesiva un factor de producción para incentivar una producción adicional, acaba disminuyendo cuando se mantienen constante otros factores.

Para Rosetti (2002), el factor capital implica considerar todas las riquezas acumuladas por la sociedad y con su empleo, la población activa se abastece para llevar a cabo sus actividades de producción, mediante un proceso de acumulación que también se destinan a la producción de nuevas riquezas materiales y no materiales y entre las principales categorías de bienes de capital que las sociedad modernas emplean en el proceso de producción se mencionan las siguientes:

- Infraestructura económica: Desarrollo de sistemas para la generación, transmisión y distribución de energía, telecomunicaciones y transportes.

\section{El papel de innovación y el cambio tecnológico}


- Infraestructura Social: Sistemas de saneamiento básico, tratamiento de aguas y otros rubros de carácter social como educación, cultura, deportes, etc.

- $\quad$ Construcciones y edificaciones: Inmuebles destinados para fines industriales, comerciales, habitacionales y militares.

- $\quad$ Equipos de transporte: Bienes de capital empleados en el proceso de producción como aviones, camiones, embarcaciones.

- Máquinas, equipos, instrumentos y herramientas: Tecnologías utilizadas en la transformación de materias primas durante la generación de actividades de prestación de servicios públicos y privados.

- Agrocapitales: Cultivos permanentes, crías de animales, presas, edificaciones.

La ampliación de capital ha sido un factor que ha repercutido de manera directa en la capacidad productiva del sector industrial, ya que dicha ampliación implicó la concesión del equipo necesario a la mano de obra, aunque al mismo tiempo, al intentar aumentar la productividad, se ha exigido acumular de forma lenta las grandes existencias de capital para cada persona económicamente activa y por lo tanto, la inversión que aumenta el capital por trabajador es, y probablemente se mantendrá como una de las influencias más eficaces para acrecentar constantemente la producción por trabajador (Heilbroner, 1981).

\section{Velázquez Juárez, J.}


Al momento de explicar la forma en que la acumulación de capital ha transformado la productividad en los sectores productivos y en las actividades económicas de los países mediante la innovación tecnológica, se refiere principalmente a la introducción de nuevos tipos de inventos que incentivan la creación de demanda y que al mismo tiempo también sustituyen mano de obra, lo cual, conlleva a que se busque con mayor intensidad, la generación de nuevos productos y procesos, que constituyan el factor principal que sustente las inversiones aplicadas para acrecentar la productividad.

Si bien se discute la importancia que tiene la aplicación de inversiones en tecnología y acumulación de capital como un simple medio de incrementar cantidades de capital a la fuerza de trabajo, sigue teniendo una importancia muy amplia, especialmente como transmisora del cambio tecnológico en una región, país o sector industrial, especialmente, aquel que se origine de la aplicación de conocimientos científicos; sin embargo, es relevante saber si la sociedad industrial tendrá la capacidad de mantener o igualar los niveles de crecimiento e innovación tecnológica.

Desarrollo y aplicación de capacidades tecnológicas como nexo entre los factores de producción

Este concepto guarda una relación muy cercana con el desarrollo de conocimientos y habilidades que sustentan al proceso de producción, abarcando desde los conocimientos acumulados de las fuentes de energías empleadas, extracción y ubicación de nuevas fuentes de reservas naturales, su procesamiento, transformación y reciclaje, hasta

\section{El papel de innovación y el cambio tecnológico}


su configuración y desempeño de los productos finales resultantes, involucrando al proceso productivo en todas sus etapas (Rosetti, 2002).

Para los franceses, el concepto de tecnología se resume en la expresión savoir faire y que corresponde a la expresión inglesa know how; para ello, es importante considerar que la tecnología concentra todos los conocimientos y habilidades se saber hacer y de cómo hacer, y que se transmiten de generación en generación y que se modifica en la dirección de formas operacionales de complejidad creciente, desde los primeros conocimientos dominados por el hombre como la rueda y el fuego hasta los más recientes avances resultantes del binomio conformado por la investigación y el desarrollo.

Convencionalmente, las habilidades y los conocimientos que son utilizados para la innovación tecnológica, implican que las nuevas habilidades o conocimientos acumulados se irán incorporando de forma simultánea a los bienes de capital y al conjunto de capacidades de la fuerza laboral, integrando de manera racional las capacidades para almacenar, procesar, interpretar y fusionar conocimientos técnicocientíficos.

Con la intención de mostrar el alcance y la descripción que tienen las capacidades tecnológicas como una serie de conocimientos y habilidades que funcionan como un nexo entre los principales factores de producción (tierra, trabajo y capital), se muestra en la tabla 1, una serie de conceptos y tipologías sobre la generación y aplicación de capacidades tecnológicas y sus principales rasgos.

Velázquez Juárez, J. 
Tabla 1. Capacidades tecnológicas: conceptos y tipología

\begin{tabular}{|c|c|}
\hline Capacidad Tecnológica & $\begin{array}{l}\text { Conjunto de conocimientos y habilidades que sustentan a } \\
\text { los procesos de producción y que corresponden a las } \\
\text { expresiones savoir faire (saber hacer) o know-how } \\
\text { (cómo hacer). Se encuentra por toda la cadena } \\
\text { productiva y está presente en todos los sectores y } \\
\text { actividades humanas de producción. }\end{array}$ \\
\hline $\begin{array}{l}\text { Tipo de capacidad } \\
\text { Tecnológica }\end{array}$ & $\begin{array}{l}\text { Generación de actividades de investigación y desarrollo } \\
(I+D) \\
\text { Creación e implementación de nuevos proyectos } \\
\text { Realización de actividades de producción }\end{array}$ \\
\hline Capacitación para I+D & $\begin{array}{l}\text { Es la aplicación de talento, conocimiento y habilidades } \\
\text { requeridas para actividades de investigación básica y } \\
\text { aplicada, donde se abarcan tecnologías de } \\
\text { almacenamiento, procesamiento, interpretación, fusión e } \\
\text { interacción de conocimiento técnico-científicos y se } \\
\text { deriva fundamentalmente en invenciones y } \\
\text { descubrimientos. }\end{array}$ \\
\hline $\begin{array}{l}\text { Capacitación para } \\
\text { desarrollo e implantación } \\
\text { de proyectos }\end{array}$ & $\begin{array}{l}\text { Representan todos los conocimientos y habilidades } \\
\text { necesarias para el diseño de proyectos relacionados con } \\
\text { nuevos procesos y productos, incluyendo la elección y } \\
\text { combinación de tecnologías dominadas y de nueva } \\
\text { generación y que sirven para definir plantas y hacer } \\
\text { viable la producción de prototipos en escala económica, } \\
\text { siendo el paso del descubrimiento a la innovación. }\end{array}$ \\
\hline $\begin{array}{l}\text { Capacitación para operar } \\
\text { el proceso de producción }\end{array}$ & $\begin{array}{l}\text { Son todas las capacidades asociadas a la operación del } \\
\text { proceso productivo, abarcando actividades relacionadas } \\
\text { con el mantenimiento de plantas, planeación y control de } \\
\text { producción, optimización de procesos y control de calidad } \\
\text { de los productos resultantes. }\end{array}$ \\
\hline
\end{tabular}

Fuente: Rosetti (2002).

El papel de innovación y el cambio tecnológico 
La evolución del conocimiento tecnológico acumulado es el resultado originado de los recursos que se destinan a la investigación y desarrollo (l+D), a nivel básico y aplicado, donde el primero representa primordialmente, un interés cientifico aunque no siempre finalice en aplicaciones de resultados económicos inmediatos y que fundamenta a los desarrollos derivados y que da origen a la investigación aplicada.

Sin embargo, entre estos dos campos de investigación se desarrollan relaciones biunívocas de naturaleza semejante a las que se registran entre los desarrollos principales y los derivados de ellos en el campo en que se experimenta la transposición de investigaciones en innovaciones. Para ello, se describe en la figura 1, los procesos de generación de acumulación y de retroalimentación de la capacidad tecnológica que facilitarán la descripción de lo mencionado previamente.

Figura 1. Procesos de generación, acumulación y retroalimentación de la capacidad tecnológica

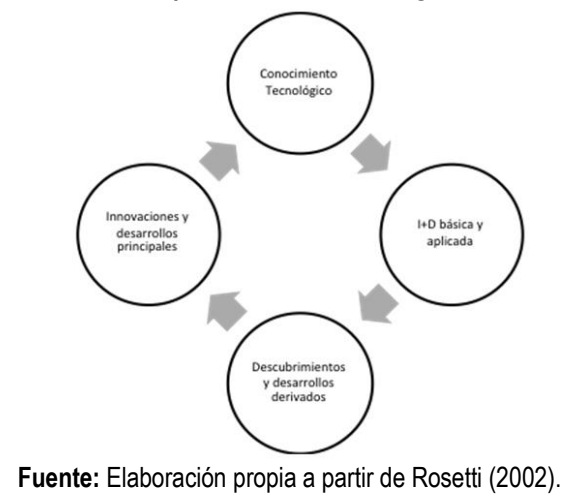

Velázquez Juárez, J. 
Los procesos de generación, acumulación y retroalimentación de las capacidades tecnológicas normalmente tienen un efecto muy fuerte en la modificación y en el desarrollo de los productos finales llevados a los mercados de consumo, además de los bienes de capital empleados para producirlos y las formas de organización y operación de las unidades de producción.

Los nuevos patrones tecnológicos se materializan bajo la forma de nueva maquinaria y equipo, de nuevas técnicas de organización de la producción y de nuevos productos finales, que dan explicación a la expansión económica.

Los avances tecnológicos han asumido una importancia similar o mayor respecto a otros factores de producción como las reservas naturales, la acumulación de capital y la existencia del grupo de la fuerza de trabajo, como una manera de explicar las modificaciones en el crecimiento económico de una región o país, principalmente como una manifestación del proceso de desarrollo, incorporación de nuevas técnicas productivas que no solo están destinadas a la acumulación de capital, aunque la incorporación de innovaciones tecnológicas implique un uso más intensivo de este.

Debido a que la técnica representa una manifestación física en el capital, el uso de nuevas tecnologías acelera la incorporación de esta, y por ello, no basta la capacidad de generar excedentes para acelerar el desarrollo, pues éste solo se realizará cuando el excedente se reincorpore al sistema productivo en la conformación de nuevas combinaciones tecnológicas, esto es, en la formación de capital de tipo

\section{El papel de innovación y el cambio tecnológico}


esencialmente distinto del que predomina en las actividades industriales y económicas.

Los resultados y los recursos aplicados a este proceso de creación de capacidades tecnológicas se han reflejado principalmente en recursos aplicados a la investigación y desarrollo, el número de instituciones y personas involucradas en estas actividades, la cantidad de invenciones patentadas y protegidas por los investigadores y científicos nacionales, así como los contratos de permisos y de absorción de tecnología desarrollada en otros países.

Al mismo tiempo, el número de innovaciones generadas se ha visto reflejada por la cantidad de productos que se ofrecen en el mercado, por la velocidad de cambio en los materiales empleados, en los procesos adoptados y en los productos resultantes, así como por las innovaciones que la sociedad asimila de manera efectiva.

Para mostrar la manera en que los avances asimilados e incorporados al acervo de la capacidad tecnológica de los países impulsan el desarrollo tecnológico, se muestra en la tabla 2, las fuentes y los resultados aparentes del proceso de desarrollo tecnológico.

Tabla 2. Fuentes y resultados generados del proceso de desarrollo tecnológico 


Gastos en I+D
Número de instituciones implicadas en
procesos de $I+D$
Invenciones patentadas

Número de personas involucradas en actividades de $1+D$
Número de innovaciones introducidas en el mercado

Velocidad de los cambios en materiales, procesos y productos

Innovaciones y cambios efectivamente asimilados.

Contratos de permisos firmados

Fuente: Tomado de Rosetti (2002).

El cambio tecnológico endógeno y su rol en la generación de crecimiento económico

La teoría del crecimiento económico ha empezado de manera constante a incorporar el papel que tiene la modificación del cambio tecnológico, ya que los efectos de un incremento en todas las empresas de sus insumos trabajo y capital tienen un efecto inmediato en los niveles de producción, donde por lo general muestran un rendimiento creciente a escala, al considerar los efectos que los incrementos de capital y trabajo tienen en la tecnología (Froyen, 1997).

Desde la Revolución Industrial, el cambio tecnológico tuvo un avance muy acelerado desde la aparición de las nuevas técnicas de hilar y tejer, las cuales fueron cruciales, mientras que, en Estados Unidos, el rápido desarrollo y difusión de la tecnología se reflejó a través de la generación y difusión de tecnologías de semiconductores. Asimismo, el crecimiento que no puede explicarse por adiciones en la cantidad de insumos sólo se explica por un incremento de la productividad de éstos, en la que cada unidad de insumo debe producir más. La productividad de un insumo resiente el efecto de factores como

\section{El papel de innovación y el cambio tecnológico}


el cambio tecnológico, otros avances del conocimiento y economías de escala (Case y Fair, 2008).

La productividad se ve afectada por el cambio tecnológico en dos etapas, donde en la primera hay un avance en los conocimientos, una invención; sin embargo, los conocimientos en sí no hacen nada si no se utilizan y al aplicar dichos nuevos conocimientos a la elaboración de un producto nuevo o para fabricar con mayor eficiencia uno que ya existe, se puede mencionar que ya hay una innovación.

Por otra parte, el cambio tecnológico no puede medirse directamente y en algunos estudios se han presentado datos sobre "indicadores" de protección de invenciones (por ejemplo, el número de nuevas patentes), pero ninguno es satisfactorio, ya que realmente no se muestra cual está siendo el avance real en la generación de innovaciones tecnológicas.

Ciclos económicos y su relación con la innovación tecnológica

Samuelson et al (2001) definen que un ciclo económico es una oscilación de la producción, el ingreso y el empleo de todo el país que suele durar de 2 a 10 años y que se caracteriza por una expansión 0 una contracción general de la mayoría de los sectores de la economía de un país.

Samuelson et al. (2001) señalan que los ciclos económicos se dividen en dos grandes fases: la recesión y la expansión, donde por lo general, la primera implica una recesión y es un periodo en que la producción disminuye durante al menos dos trimestres consecutivos y la

\section{Velázquez Juárez, J.}


expansión por lo general comprende el efecto contrario a la recesión y es un periodo en el que la producción se incrementa durante al menos dos trimestres consecutivos.

Dornbusch, Fischer y Startz (2015) explican que un ciclo económico es un patrón más 0 menos regular de expansión (recuperación) y contracción (recesión) de la actividad económica respecto a la línea de tendencia de crecimiento, donde en un pico del ciclo, la actividad económica es elevada respecto a la tendencia (Auge), mientras que en el fondo del ciclo, se llega al punto más bajo de la actividad económica (depresión); por lo tanto, la inflación y el crecimiento cuentan con patrones cíclicos claros, aunque fluctúa constantemente alrededor de esa tendencia.

Dornbusch et al. (2015) agregan que la producción no tiene siempre un nivel tendencial que corresponda al pleno empleo de los factores de producción, sin embargo, al haber una expansión 0 recuperación de una economía, el empleo de los factores de producción aumenta, lo cual, se refleja en el incremento de la producción.

Rosseti (2002), explica que los ciclos económicos y las fases de prosperidad se atribuyen principalmente a los segmentos de invención e innovación en tecnologías de proceso y producción, donde el indicador clave en esta relación pasa por detectar los ciclos de vida de las innovaciones desarrolladas en determinada época, ya que de esta manera, la prosperidad está asegurada, aunque pudiera llegar a ser interrumpida por movimientos de contracción de menor amplitud y de corta duración.

\section{El papel de innovación y el cambio tecnológico}


Emprendedurismo y capacidad empresarial

La capacidad empresarial representa uno de los elementos que conforma a los factores de producción de las economías nacionales, que tiene un papel donde aprovecha que el descubrimiento y la explotación de recursos naturales, los cambios demográficos, la selección de bienes de capital, la definición de patrones tecnológicos a emplear y el desplazamiento, aglutinación y combinación de los diferentes factores de producción, implican una transformación a través de un sector industrial (Samuelson et al., 2001).

La capacidad empresarial se manifiesta al ser empleada de manera conjunta y de su aglutinación alrededor de un determinado proyecto, su adecuada combinación, así como la organización y dirección a que se someten, resultando en flujos de producción, aprovechando los factores de producción previamente mencionados.

Por otra parte, el término emprendedor proviene del vocablo francés entrepreneur y abarcaba originalmente las funciones de inventor, planificador, constructor, administrador y patrón, aunque posteriormente empezó a incorporar sus funciones como proveedor de capital y administrador de riesgos, como una forma de obtener utilidades a través de la ejecución de acciones.

Rosetti (2002) señala que el emprendedurismo y la capacidad empresarial cuentan con una serie de cualidades que los diferencian de los diferentes unidades económicas y entre las principales características del emprendedurismo y la capacidad empresarial más comunes se destaca la visión estratégica orientada al largo plazo, la

Velázquez Juárez, J. 
medición y control de riesgos, la capacidad para innovar, cambiar paradigmas y proponer soluciones a problemas de las personas, la sensibilidad para detectar posibles oportunidades de inversión, así como la disponibilidad para acceder a los cuatro factores de producción, además de la capacidad para combinarlos y motivarlos, contando con las habilidades y competencias para organizar proyectos, adquiriendo 0 contratando los factores necesarios, delegando de ser posible diversas actividades administrativas.

Innovación tecnológica

Valderrama, Neme y Ángeles (2016) explican que la innovación es un proceso complejo de creación de novedades con significado económico en el que intervienen diversos agentes como empresas, organizaciones públicas y privadas, instituciones educativas entre otros que también se encargan de facilitar la generación, difusión e implementación de la innovación.

Valderrama et al. (2016) señalan que la innovación es un valor reconocido en el mundo empresarial, por lo que cada vez más organizaciones tienden a considerar prácticas, procesos e instrumentos para crear una cultura de innovación que les contribuya a mejorar sus resultados, llevando al mercado diversos productos, servicios procesos, conceptos, ideas o proyectos que son aceptados por los clientes, con la finalidad de satisfacer las necesidades de las personas, otorgando un valor agregado a la oferta disponible.

Castellanos (2007) explica que la innovación representa una variable que cobra una gran importancia en el desarrollo de una

\section{El papel de innovación y el cambio tecnológico}


organización, ya que puede facilitar las posibilidades de generar novedosos cambios en procesos productivos y desarrollo de productos, a partir del comportamiento pasado y presente del proceso y de las tendencias que presenten los mercados, así como la variación de factores que pueden afectar en dichos patrones como el volumen de ventas y la evolución del comportamiento del consumidor.

Por otra parte, Ramos, Urbiola, y González (2014) explican que la innovación tecnológica representa un proceso de transformación 0 cambio que suele ocurrir en un proceso industrial y que permite modificar la forma de concebir, ejecutar y comercializar productos 0 servicios.

Sin embargo, Ramos et al. (2014) agregan que la innovación se ha convertido en un proceso más complejo por la diversidad de inputs manejados por los actores económicos, además de que su creciente interdependencia y división de trabajo altamente especializado requiere de un manejo adecuado a nivel científico y social, pertinente para el mercado de productos tecnológicos.

García (2007) definen que la innovación tecnológica comprende un proceso sistemático y deliberado que modifica determinados factores que inciden en la generación de riqueza o una nueva capacidad antes que conocimientos tecnológicos o científicos, aunque se requieran de igual forma la capacidad para interrogar y descubrir la realidad, así como la cualidad de romper prejuicios y abrirse al cambio.

García (2007) explica que una innovación tecnológica comprende tres momentos, cuyo comienzo se da de partir de una idea proveniente de un descubridor capaz de interpretar la situación desde

Velázquez Juárez, J. 
un ángulo diferentes, brindando nuevas visiones, para posteriormente aterrizarla y desde la realidad actual, ejecutarla y finalmente, la idea se implante, difundiendo y aprovechando su desarrollo masivamente.

Con la finalidad de explicar con mayor facilidad este concepto, se presenta en la figura 2 , el procedimiento común que sigue el proceso de la innovación tecnológica.

Figura 2. Procesos de la innovación tecnológica

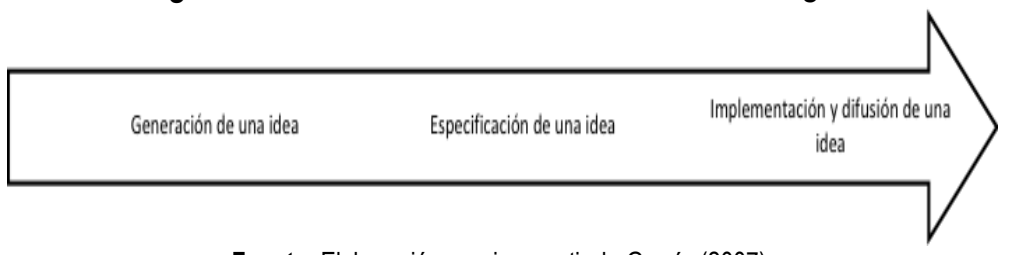

Fuente: Elaboración propia a partir de García (2007).

Clasificación de la innovación conforme a su naturaleza

García (2007) sostiene que las innovaciones se clasifican de múltiples maneras y con la intención de brindar elementos que permitan delimitar los requerimientos intelectuales para cada una de ellas en función de sus rasgos, se muestra en la tabla 3 , se muestra una clasificación general de dichos tipos de innovaciones.

Tabla 3. Clasificación de las innovaciones

$\begin{array}{ll}\text { Tipo de innovación } & \text { Características y rasgos principales } \\ \text { Tecnológica } & \text { Generación de nuevos artefactos a través de productos, } \\ & \text { servicios o procesos novedosos. Se relaciona con la planta } \\ & \text { productiva e ingeniería }\end{array}$

El papel de innovación y el cambio tecnológico 
Comercial Desarrollo de nuevas estrategias de promoción, medios de distribución y comercialización de productos y servicios.

Organizacional Se centra en el diseño y organización de tareas, estilo de dirección y aprovechamiento de capital humano. Se vincula a los ámbitos de dirección empresarial, administración y recursos humanos

Financiera $\quad$ Se refiere a nuevas maneras de administrar y manejar recursos monetarios, además de la generación de nuevos instrumentos bursátiles Se relaciona con las áreas de tesorería, administración financiera y mercado de dinero.

Servicios Se refiere a maneras de actualizar o mejorar la prestación de un bien o servicio de cualquier índole, ya sea de carácter educativo, de salud, turístico, asesoría, etcétera.

Fuente: Elaboración propia a partir de García (2007).

Davenport, Leibold y Voelpel (2006) citado en Velázquez, Valencia y Peña (2016), sostienen que se cuenta con una clasificación de carácter amplia y confusa para describir el concepto de innovación y que se ubica principalmente en los avances de la organización industrial.

De esta forma, se puede sostener que la innovación representa un vector que impulsa la generación de avances tecnológicos, manifestándose a través de la creación de nuevos productos, así como en la habilidad de elaborar los ya existentes a menor costo, siendo uno de los motores más importantes que impulsen el crecimiento económico.

Con la intención de mostrar de manera general la clasificación de la innovación, se muestra en la tabla 4 los diferentes ángulos de estudio sobre la innovación, destacando su multidimensionalidad y

\section{Velázquez Juárez, J.}


diversidad de enfoques.

Tabla 4. Clasificación de Innovaciones tecnológicas

\begin{tabular}{|c|c|}
\hline $\begin{array}{c}\text { Tipo de } \\
\text { Innovación }\end{array}$ & Clasificación \\
\hline $\begin{array}{l}\text { Innovación } \\
\text { disruptiva }\end{array}$ & $\begin{array}{l}\text { Son innovaciones que surgen de la nada, crean nuevas fuentes } \\
\text { masivas de ganancias y surgen comúnmente de discontinuidades } \\
\text { tecnológicas. }\end{array}$ \\
\hline $\begin{array}{l}\text { Innovación } \\
\text { aplicada }\end{array}$ & $\begin{array}{l}\text { Se desarrollan a partir de tecnologías existentes y son dirigidas } \\
\text { hacia nuevos mercados, que a su vez, buscan ser utilizados en } \\
\text { nuevas aplicaciones, que den lugar a crear nuevos productos y } \\
\text { servicios. }\end{array}$ \\
\hline $\begin{array}{l}\text { Innovación en } \\
\text { productos }\end{array}$ & $\begin{array}{l}\text { Este tipo de innovación se da en mercados establecidos y son } \\
\text { llevados a un nivel más elevado y se enfocan en mejorar su uso, } \\
\text { disminuir costos de producción y mejorar su rendimiento. }\end{array}$ \\
\hline $\begin{array}{l}\text { Innovación en } \\
\text { procesos: }\end{array}$ & $\begin{array}{l}\text { Utiliza nuevos procesos para ser aplicados en productos } \\
\text { disponibles dentro de mercados establecidos con la finalidad de } \\
\text { mejorar su eficiencia y efectividad. }\end{array}$ \\
\hline
\end{tabular}

Escorsa y Valls (2005), explican que los resultados de la innovación tecnológica se pueden presentar de diversas formas tales como cambios radicales 0 incrementales, modificaciones totales de elementos existentes o creación de nuevos elementos, nuevas opciones incorporadas a productos, procesos 0 servicios, orientados al consumidor, la industria y/o el gobierno, basados en una sola 0 en múltiples tecnologías. La innovación por lo general tiene su primer uso en manufactura de procesos o en el mercado.

Escorsa y Valls (2005) agregan que se deben distinguir dos

\section{El papel de innovación y el cambio tecnológico}


tipos de innovaciones: revolucionaria y progresiva. Una innovación revolucionaria es aquella capaz de crear un mercado nuevo o de modificar sustancialmente la estructura competitiva de uno ya existente, dando la oportunidad de crear ventajas competitivas en aquellas organizaciones que la poseen. Este caso se presenta comúnmente en computadoras personales, teléfonos celulares y tabletas.

Ahmed Ramos y Ramos (2012), argumentan que la innovación suele manifestarse de múltiples formas y maneras, tales como la innovación estratégica, innovación en productos y en procesos, que surgen de los diferentes significados adscritos, pero que, de manera general, son identificados a partir de las habilidades de una empresa para ser empleados en múltiples actividades. Dentro de la figura 3, se muestran las manifestaciones más comunes de la innovación, a partir de las capacidades de la empresa para controlar y clasificar dichas novedades.

Figura 3. Tipos de innovación
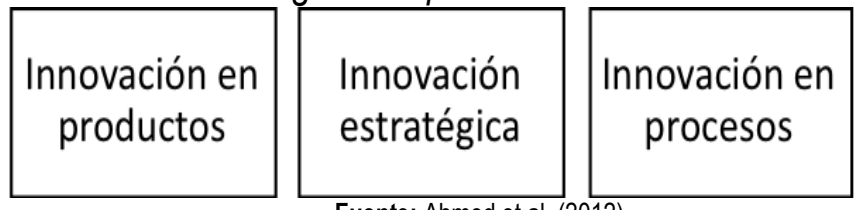

Fuente: Ahmed et al. (2012).

Afuah (1997) destaca que la innovación tecnológica puede clasificarse en radical y en incremental, donde la primera se manifiesta en un producto que es tan superior (menor costo, mejores atributos 0 nuevos atributos) que los productos existentes se vuelven no

Velázquez Juárez, J. 
competitivos, mientras que la segunda representa solamente cambios menores o graduales en la estructura del producto, conservando competitividad y manteniendo precios estables o más bajos.

Afuah (1997) recalca que la clasificación propuesta para la descripción de las innovaciones tecnológicas, repercuten en la estructura organizacional y en la competitividad y en los incentivos estratégicos para invertir en tecnología, además de las capacidades de explotar adecuadamente las tecnologías sin obstaculizar sus procedimientos ya definidos.

\section{Conclusión}

La incorporación de cambios tecnológicos desde la perspectiva de la macroeconomía permite producir una mayor cantidad de bienes y servicios con la misma cantidad de productos, considerando también que el cambio tecnológico ha tenido un papel muy importante al impulsar actividades económicas y en consecuencia, el crecimiento económico y la mejora de la calidad de vida.

La velocidad con la que un país incorpora nuevos inventos o nuevos procedimientos en su sistema productivo, esto es la velocidad en que aparecen los cambios tecnológicos, están fuertemente ligados a los recursos que se destinan a las actividades de investigación, desarrollo e innovación (I+D+i) y la forma en que se gestionan dichos recursos.

La investigación permite desarrollar nuevo conocimiento, mientras que el desarrollo se utiliza para cuando los conocimientos creados se van transformando y adaptando para ser aplicables en la producción y

\section{El papel de innovación y el cambio tecnológico}


finalmente, la innovación conlleva a aplicar nuevos conocimientos, inventos, técnicas o procedimientos a la producción.

Al hablar del papel de la economía dentro del cambio tecnológico, es necesario también considerar que si un avance tecnológico requiere la instalación de nuevo capital físico, es decir, maquinaria, se dice que se trata de una tecnología incorporada en el capital. Asimismo, desde el enfoque económico, el crecimiento representa una serie de procesos generadores de progreso tecnológico, los cuales consideran principalmente la acumulación de capital humano por parte de los trabajadores y la investigación y desarrollo de nuevos productos y procesos productivos (tecnología nueva) por parte de las empresas.

Asimismo, para ver de mejor manera los resultados obtenidos de la innovación tecnológica, se deben considerar la aplicación de grandes cantidad de recursos, los cuales pueden ser al mismo tiempo, muy fáciles de replicar y por ello, la creación y transmisión de innovaciones y de información resulta muy difícil de asegurar, pues suele verse afecta por riesgos difíciles de controlas, además de que el inventor procurará proveer en exclusiva su invención, pero al mismo tiempo, resultará difícil de imitar.

\section{Referencias}

Afuah, A. (1997). La dinámica de la innovación organizacional. Nueva York: Oxford Ahmed, P., Ramos, L., \& Ramos, C. (2012). Administración de la Innovación. México: Pearson Educación

Velázquez Juárez, J. 
Case, K., y Fair, R. (2008). Principios de Macroeconomía. Ciudad de México: Pearson Educación

Castellanos, O. (2007). Gestión Tecnológica. Medellín: Universidad Nacional de Colombia

Clement, D. J. (1997). U.S. Patent No. 5,684,963. Patent and Trademark Office. Washington, DC: U.S. Disponible en: https://patents.google.com/patent/US5684963A/en

Davenport, T. H., Leibold, M., y Voelpel, S. (2006). Strategic Management in the Innovation Economy. Erlangen: Publicis

Dornbusch, R., Fischer, S., y Startz, R. (2015). Macroeconomía. México: McGraw Hill

Escorsa, P., y Valls, J. (2005). Tecnología e Innovación en la empresa. Barcelona: Alfaomega

Froyen, R. T. (1997). Macroeconomía: Teorías y políticas. México: Prentice Hall

García, F. (2007). La Investigación Tecnológica: investigar, idear e innovar en ingenierías y Ciencias Sociales. México: Limusa

Heilbroner, R. (1981). Comprensión de la Macroeconomía. México: Uteha

Parkin, M., y Loria, E. (2010). Macroeconomía: versión para Latinoamérica. México: Pearson Educación

Ramos, N., Urbiola, A. y González, E. (2014). La gestión de las innovaciones tecnológicas y su manifestación. Gestión, tecnología e innovación. México: Pearson, 3-18

Rosetti, J. (2002). Introducción a la Economía. Sao Paulo: Alfaomega

Samuelson, P. A., Nordhaus, W. D., Dieck, L., y Salazar, J. d. (2001). Macroeconomía con aplicaciones a México. México: Mcgraw Hill

Samuelson, P., y Nordhaus, W. (2006). Economía. Ciudad de México: McGraw Hill

Valderrama, A., Neme, O., y Ángeles, G. (2016). Diagnóstico de habilidades de innovación en lasmicro y pequeñas empresas en México: El individuo Innovador. Ciudad de México: Colofón

Velázquez, A., Valencia, R., y Peña, J. (2016). El papel del modelo de la triple hélice como sistema de innovación para aumentar la rentabilidad en una Pyme comercializadora. Revista Ciencias Económicas y Administrativas, 2(3). 101-112

\section{El papel de innovación y el cambio tecnológico}

